\title{
Content server scalability
}

\section{Michael Blakeley}

is a principal consultant at Mark Logic Corporation, where he specializes in performance and scalability. His prior career includes performance work at Netscape Communications, Silicon Graphics and PC Week (now eWeek), as well as performance-related consulting engagements for numerous clients.

Keywords: DAM, XML, XQuery, custom publishing, scalability, performance

Abstract The growing size and complexity of content makes it increasingly difficult for content management systems and content-based applications to keep up. Fast access to terabytes (TB) of eXtensible Markup Language is increasingly important to many companies and organizations. Filesystems and relational database management systems are adequate for gigabytes of rich content, but TBs demand a repository that was built for content.

Journal of Digital Asset Management (2007) 3, 239-245. doi:10.1057/palgrave.dam.3650092

\section{INTRODUCTION}

Many companies need to manage large quantities of digital assets. Large scientific publishers, government agencies, pharmaceutical companies, airlines, regulated utilities... the list goes on and on. These companies produce or manage hundreds of gigabytes (GB) to terabytes (TB) of content every year. Increasingly, this content is represented as XML (eXtensible Markup Language).

Migrating legacy document formats to XML is a huge, and sometimes Sisyphean task. But once you have all that XML, where do you put it? Historically, content publishers have stored content on filesystems and in RDBMS tables (Relational Database Management System). Some have used search engines to index the content, so that they can find important documents more quickly. Some have built RDBMS functional-indexes around their XML content, or shredded the XML into relational columns and rows.

As content repositories grow, these solutions are not good enough. Applications built around monolithic documents do not provide sufficient ROI to justify the cost of converting to XML, and customers - whether internal or external Mark Logic Corporation, 999 Skyway Road, Suite 200, San Carlos, CA 94070 , USA

Tel: +1 6506552300 Fax: +16506552310 Email: michael.blakeley@ marklogic.com
- are not satisfied with the results. Whether

you call it Web 2.0 or not, customers

increasingly want to blend documents together.

They want to create their own content products and be their own editors. This trend toward "custom publishing" (or reuse of rich content) can be seen in many information-driven businesses.

\section{CUSTOM PUBLISHING AND ITS CHALLENGES}

An example of this kind of application is O'Reilly Publishing's SafariU (https://www. safariu.com/). SafariU lets educators mix their own textbooks by selecting chapters from existing textbooks. ${ }^{1}$ Students can then buy custom-published copies of these textbooks. This kind of application disrupts traditional publishing models in much the same way that Apple's iTunes has disrupted the CD-based music publishing model.

Custom publishing, though, is not limited to traditional publishers. Many companies publish large quantities of documents that can benefit from a custom-publishing approach. Financial analysts, for example, can create custom reports by mixing components from existing documents with newly created content. Airlines can publish flight manuals for their pilots by mixing documentation from Boeing or Airbus with internally created content — some general purpose, and some restricted to a specific model of airplane or a specific market. Finally, intelligence work often lends itself to some form of custom publishing.

Custom publishing applications all share certain traits. They are driven by users as much as by editors. They are driven by the value of 
particular pieces of content to particular users.

And they present difficult challenges to

traditional publishing models and traditional publishing tools. $^{2}$

Some of these challenges stem from the nature of content. Relational databases have been around for years, and have made a huge impact on computing because of their ability to manipulate values in columns, rows and tables. If your information can be expressed as data that is, if it fits into columns and rows - that's great. But much of the work around content, especially the reusable content that is crucial to custom publishing, centers on hierarchy instead. When we look at a document, or a portion of a document, we do not see a table. Instead of looking like tables of data, content looks like a tree.

Content as a tree creates several problems for an RDBMS. Creating hierarchical relationships between rows is often clumsy and slow, and generally does not scale to deep levels of nesting or large numbers of rows and tables. Worse, all queries have to be written in SQL, which was not designed for the job. As Stonebreaker and Cetintemel argue, ${ }^{3}$ the RDBMS "one size fits all" approach is not flexible enough to build complex applications on unstructured or semi-structured content.

To address these challenges, some organizations have decided that an RDBMS simply does not add value to their content. Instead of treating the content as columns and rows in relational tables, they might decide to leave the documents on the filesystem. This creates a new challenge: how can an application enable users to access the millions, or even billions of documents in our content-store, and find the handful of documents they want to use?

Many organizations respond to this scalability problem by bolting on a search engine, which indexes the documents. But even with the best search engine technology, a filesystem-based approach loses something valuable from the database world: ACID properties. Unlike updates to the database, updates to the filesystem are not necessarily atomic, consistent, isolated and durable. This is especially important in the context of search: after every update to the filesystem, the search indexes must be updated. Depending on the technology used and the size of the content set, the filesystem and the search results may be out of sync for minutes, hours or even days.

At this point, we can see that no existing approach is well suited to building contentdriven applications with large quantities of unstructured or semi-structured content. We can also list the characteristics of an ideal content repository.

- Hierarchy-aware query language;

- Full-text query language;

- Hierarchy-aware indexes;

- Transactional full-text indexing (updated as each document is added or modified);

- ACID (atomic, consistent, isolated, durable) transactions;

- Fast ingestion;

- Fast queries;

- Scales to TB of XML content.

For a hierarchy-aware query language, XQuery is a natural fit. For the rest of these requirements, we will look at one highly scalable product - MarkLogic Server.

MarkLogic Server is an XML content server designed and developed for big, fast XQuery. In the past four years, Mark Logic Corporation has helped customers deploy increasingly big, increasingly fast XML content server applications. Some of these applications have been new customer projects, while others have expanded on existing projects.

What is "Big, Fast XQuery?" At Mark Logic, we see XML and XQuery as an ideal platform for building a new category of rich, contentdriven applications. Our implementation of XQuery, MarkLogic Server, adds full-text search to the mix, and brings high performance and broad scalability to applications, even with massive amounts of content. Many currently deployed customer applications include $100 \mathrm{~GB}$ to multiple TB of XML, and our largest customer applications are pushing into the 100-TB arena.

When developing and deploying applications around these massive content-bases, our users demand fast responses to queries of arbitrary complexity, with large numbers of concurrent users. Many applications also demand ACID compliance: that is, every insert, update and delete must be an atomic, consistent, isolated and durable transaction. 


\section{DESIGNED FOR CONTENT}

MarkLogic Server is designed to support fast queries and transactions while scaling up to $\mathrm{TB}$ of XML. To do this, the product includes several important architectural features (Figure 1).

First, every XML element and elementattribute is indexed by value. This enables fast XPath queries over hundreds to thousands of GB of XML without any special setup. In addition, a core set of full-text indexes is populated for every XML node. This enables not just word-search and phrase-search, but also complex queries based on particular terms in one element, and additional terms in other elements or element-attributes.

The content and the indexes are stored together, in a transactional repository. This is an especially nice feature for content applications, because it means that content is searchable as soon as it is inserted. The users never have to wait for indexing to complete.

This functionality is exposed through XCC, a content API for .NET and Java developers, as well as via HTTP for service-oriented architectures (SOA). There is also a WebDAV interface, which can be handy for simple editorial applications.

Each customer has different business requirements, and so each MarkLogic Server application is different. Think about an RDBMS application: a table of 20 VARCHAR(1000) columns will do different things, and perform differently, than a table of 200 VARCHAR(100) columns. In the XML Content Server world, a document with more tags and less text will behave differently from a document with more
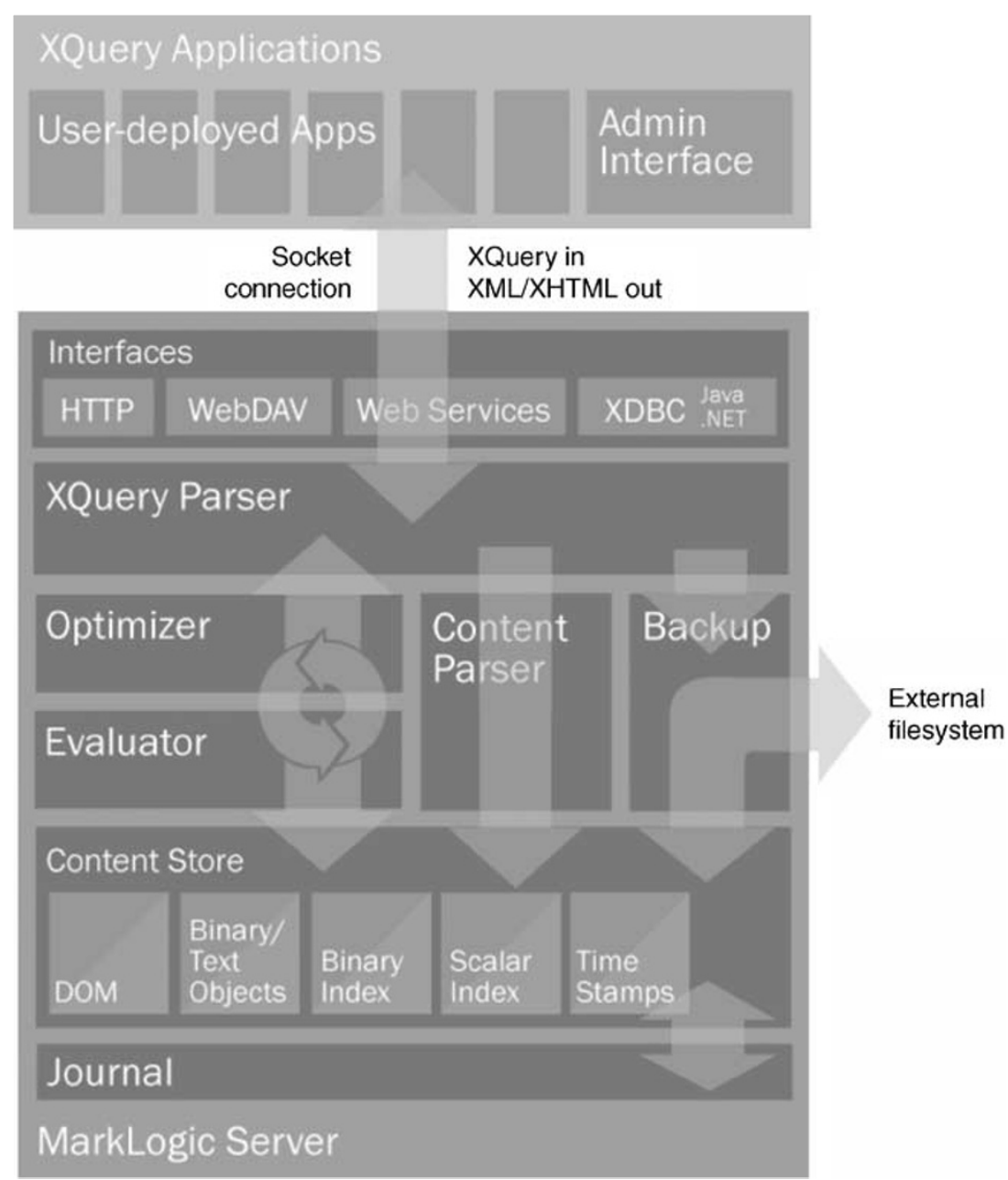

Figure 1: The internal architecture of MarkLogic Server is designed for big, fast XQuery 
text and less tags. Naturally, this affects indexing, which in turn affects and is affected by the user-submitted queries. Thus, both application content and queries will affect disk space utilization, CPU utilization and general scalability.

The variability of the content corpus is important for another reason: every text or XML document in MarkLogic Server is compressed for on-disk storage. So more compressible documents will use less disk space, even if many optional indexes are enabled (generally speaking, longer documents compress better than shorter ones do).

Besides differences in content and business requirements, we have seen growth and commoditization of computing hardware over the past three years. CPUs have become faster, cheaper and more plentiful. RAM has become cheaper and denser. Disk storage has also grown in density and fallen in price.

As a result, our sizing calculations have changed. In 2004, we might have looked at a given customer's application requirements, and recommended 2-4 CPU hosts with 8-16 GB RAM. Depending on query performance requirements, this might limit each host to $500 \mathrm{~GB}$ of MarkLogic storage per host. In 2006 we began testing dual-core CPUs and found that each performed as well as two single-core CPUs. By 2007, we were recommending 4-8 $\mathrm{CPU}$ cores per host, regardless of the number of cores per socket, with 16-32 GB RAM. For a median customer scenario, this configuration might allow up to 1TB of storage per host.

This has been, and continues to be, a great time to buy hardware. Over the past few years, 64-bit CPUs have also become less expensive. With AMD Opteron (AMD64) and Intel's newer Xeon CPUs (EM64T) widely available at commodity prices, there is no longer any pricepremium attached to 64-bit hardware. This is good for MarkLogic Server, since the architecture assumes plentiful RAM and plentiful address-space.

As components have become cheaper, we have continuously tuned our performance for a scenario where the customer spends $1 / 3$ of his server budget on CPU, 1/3 on RAM and 1/3 on storage subsystems. With good-value purchases in each category, this results in a well-balanced server (Figure 2).

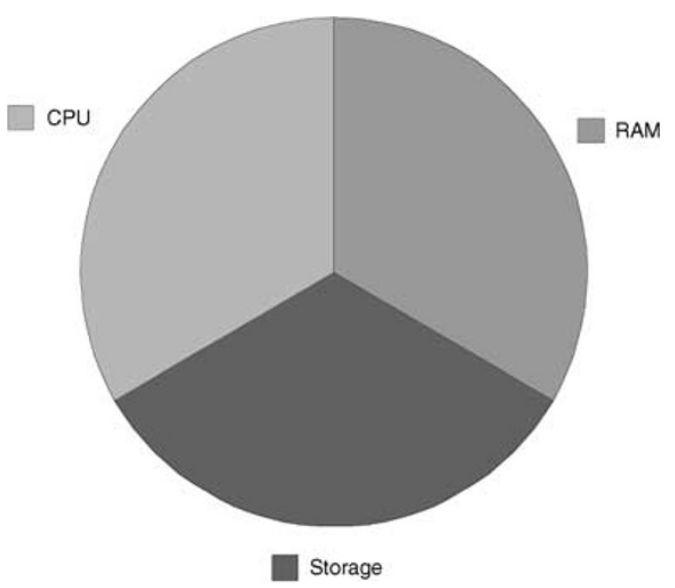

Figure 2: Ideal allocation of server hardware budget

At the moment, such a server has $4-8 \mathrm{CPU}$ cores - 64-bit, naturally. It has 16-32 GB RAM, and hosts $300 \mathrm{~GB}$ to $1 \mathrm{~TB}$ of MarkLogic storage (also called Forest space).

But so far we have not talked about scaling past 1TB. How do we get to 100TB?

As you might expect, MarkLogic Server allows multiple hosts to share a common configuration as a cluster. Within a cluster, queries are received by one of the designated evaluation hosts (E-hosts). Within a given $\mathrm{XQuery}$ module, some operations may require database operations, while others require inmemory processing. This is somewhat analogous to the interplay between SQL and PL/SQL, in Oracle's RDBMS architecture. Whenever one of these database operations is needed, the E-host sends that portion of the query to all the hosts that store content for the current database. We call those hosts D-hosts, and we call each of their distributed data storage units a Forest (Figure 3).

Another aspect of scalability is user concurrency. That is, how many simultaneous users can the system support, and how can the system grow to support more users? This becomes somewhat tricky because the notion of a "simultaneous user" is somewhat tricky. If I'm logged onto a website, but I've done nothing for five minutes, do I count as a simultaneous user? I'm probably taking up space in some session-related data structures, but I'm not using any more disk, CPU or network than I would be if I were logged out entirely. What if I do nothing for $1 \mathrm{~min}$ ? $30 \mathrm{~s}$ ? 


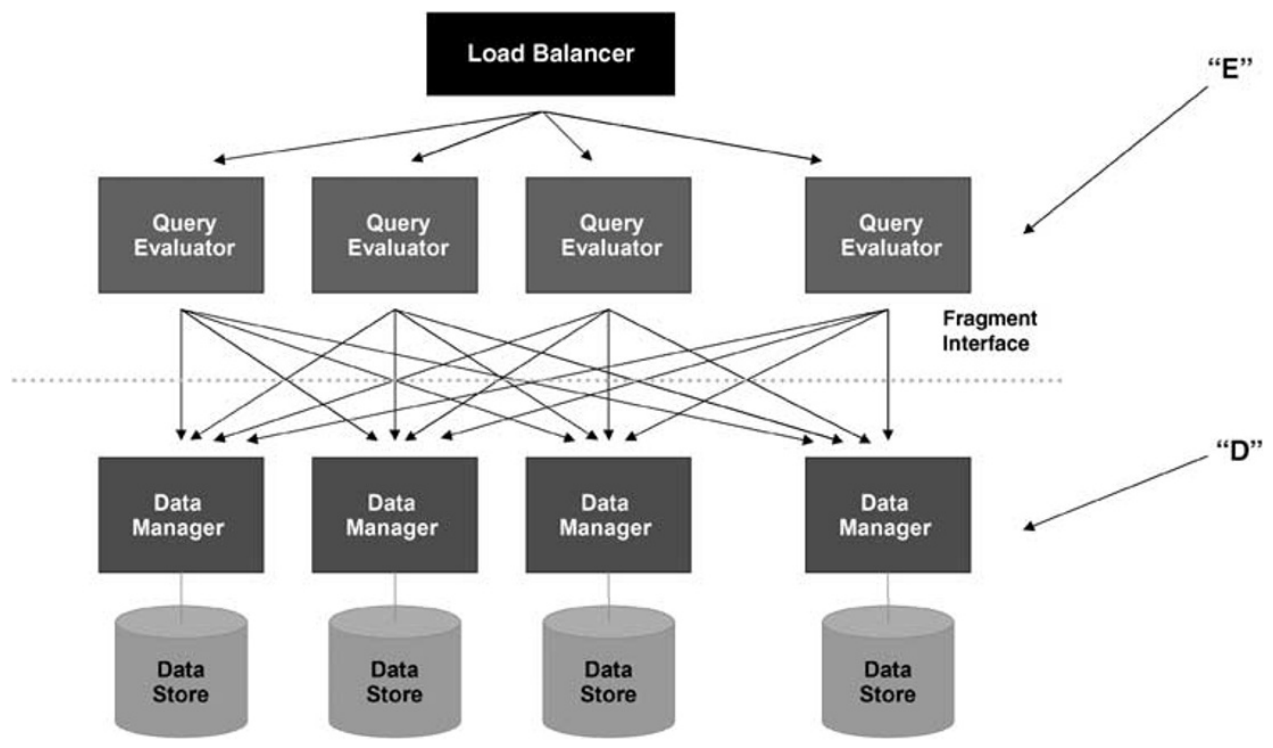

Figure 3: Architecture of a cluster of MarkLogic Server hosts, including separation between Eval and Data hosts

Instead of talking about simultaneous users, then, it is more precise to talk about query arrival rate, and the resulting number of concurrent queries. These concurrent queries are XQuery operations (point-in-time reads, or transactional updates) that are active on the cluster at the same sample-time. Essentially, a given server can have one query in flight per thread in its worker-thread pool. Optimally, a server has one query in flight per CPU core.

A single concurrent query per second might support anywhere from 1 to 1,000 logged-in users, or perhaps an entirely different number of active users, under whatever the customer's definition of "active" might be. This, too, tends to be application-specific.

We can now join up these concepts and talk about sizing in two principal dimensions: storage and concurrency. If we need to support $10 \mathrm{~TB}$ of content, we might specify 10-30 D-hosts (the exact number would depend on customer content and business requirements). If we need to support 400 concurrent queries, we might note that ten D-hosts with four CPU cores each will meet the requirements nicely.

We try to judge the amount of work that is done in the E-hosts vs the D-hosts of a cluster, for a given application. By splitting the cluster's hosts into two groups, we can tune the hosts in each group independently. For example, we might put $32 \mathrm{~GB}$ in each D-host, for better caching of database content, while specifying only $16 \mathrm{~GB}$ per E-host. We might also save money by purchasing expensive storage hardware or software for the D-hosts Fibrechannel cards, for example, or licenses for a cluster-aware filesystem.

Customers can start with one host and add additional hosts as more capacity is needed, forming a cluster. An unclustered host acts as both an E-host and a D-host. It is also possible for hosts within a cluster to play both D-host and E-host roles $(\mathrm{D}+\mathrm{E})$. This can lead to less efficient use of RAM and CPU, but D $+E$ hosts are sometimes convenient for a small cluster or for a new customer. Later on, as the cluster needs to scale up, the roles of the various hosts can be separated.

\section{CASE STUDIES}

Let us examine the performance and scalability characteristics of a few customer projects. Please note that some of our customers prefer to remain anonymous. To preserve their anonymity, we will label each project with a year. We will not mention specific companies, organizations or projects, and some technical details may be sanitized. Our website (http://marklogic.com/) has more information about selected customers. 


\section{4}

We start with an early deployment in 2004. This customer built a content-retrieval system using ten Solaris/SPARC servers, each with four UltraSPARC-IV CPUs and 32-GB RAM. All this hardware supported a total of $600 \mathrm{~GB}$ of Forests, distributed across all ten hosts. In this cluster, each server acted as both D-host and E-host $(\mathrm{D}+\mathrm{E})$.

This was the customer's first MarkLogic Server deployment, and one of our first large deployments, so we were conservative about sizing. After gaining confidence, both internally and with our customers, our sizing became somewhat more aggressive.

\section{5}

AMD brought commodity, server-class x86-64 CPUs to market with their Opteron models back in 2003, and dual-core Opterons became available in 2005. But large projects tend to be conservative, so our first large-scale deployment with Opteron CPUs chose to stick with single-core Opteron CPUs. This customer required a system that would ingest $200 \mathrm{~GB}$ of XML per day, and would support 5TB of MarkLogic storage in total.

The cluster was deployed as five D + E hosts, each running RedHat Enterprise Linux on four AMD Opteron single-core CPUs. Each host had 32-GB RAM, and a dedicated 22-disk Fibrechannel SAN using RAID-5 and SGI's XFS filesystem (Figure 4).

The completed cluster exceeded customer requirements, ingesting content at a sustained rate of $330 \mathrm{~GB}$ per day. It also sustained a high query rate in several use-cases. The figure shows performance of this cluster in terms of average response time, queries per second and client threads (concurrent queries). As we go from one

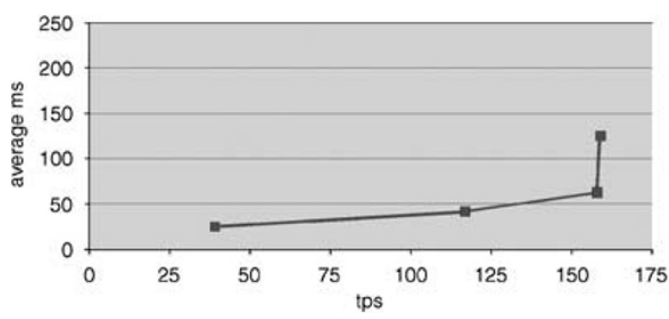

Figure 4: Each data point represents an increasing number of concurrent queries $(1,2,4$, and 8$)$. The system shows nearly ideal behavior under load client thread to two and then four threads, response time remains roughly flat and throughput increases. It is easy to see that concurrent queries are not the same as users, in this diagram: with only four concurrent queries, we sustain 160 queries per second. Real-world users might generate only 1-10 queries per minute, so this cluster could support a large number of users.

This behavior shows nearly perfect scalability from 1 to 4 threads. At four client threads we have one concurrent query running per $\mathrm{CPU}$ core on each host in the cluster (remember that every database operation checks every Forest). Adding further load does not affect the throughput, but does increase the average query response time. For optimal performance, then, we might use a load-balancer to throttle incoming load at four concurrent queries. Again, this might translate to thousands or tens of thousands of simultaneous users, depending on application requirements.

\section{6}

So far our two case-studies chose to use combined-role hosts $(\mathrm{D}+\mathrm{E})$. In 2006, another customer project needed to deploy a new MarkLogic Server application with 4TB of XML - 90 million documents. They designed their application to use some full-text search features that were introduced in MarkLogic Server 3.0: wildcards and word-positions. This extra indexing allowed their users to write much more powerful queries, but naturally had a cost in CPU time and disk space. The users of this application also demanded high query complexity: 20-100 different search terms, in Boolean combinations.

To meet the challenges of this application, we helped the customer to design a ten-host Linux cluster, using RedHat Enterprise Linux. Each host had four AMD Opteron CPUs, still single-core and 16-GB RAM per host. To take best advantage of this RAM, eight servers were designated as D-hosts and the remaining two as E-hosts. This design allowed for $500 \mathrm{~GB}$ of XML per D-host, and allowed for scalability by adding more D-hosts as the content grew, or more E-hosts as the user activity grew. Content was stored on a shared SAN, connected via dual-path Fibrechannel (Figure 5). 


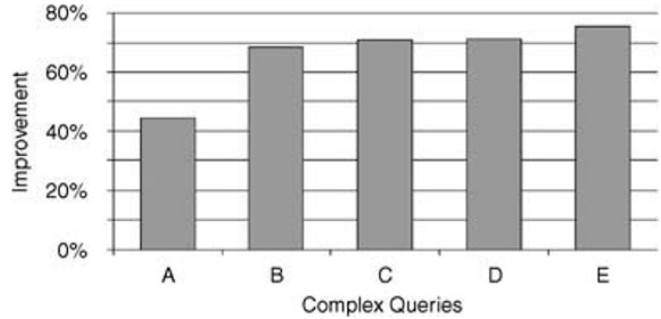

Figure 5: Queries with 20-100 terms enjoyed a significant performance boost when the customer upgraded the server software

Some time after successfully deploying this application, we released MarkLogic Server 3.1 and persuaded the customer to upgrade. This exposed another interesting facet of scalability: the engineering team had been hard at work optimizing complex queries, and so a simple software upgrade greatly improved the performance of the customer's application. The performance of individual queries improved by $40-80$ per cent, without any code changes, thus making users of the application more productive and extending the lifespan of the original cluster hardware.

\section{7}

By the start of this year, customer applications like the ones we had done in previous years had become commonplace. New customers had more aggressive goals, and one customer wanted to ingest $200 \mathrm{~TB}$ of source XML - in this case, roughly 5 billion documents. Moreover, this content would be indexed using positions and wildcards, plus several element-range indexes, and the users would generate complex queries, using up to 100 search terms in Boolean combinations (Figure 6).

The design for this cluster was our most ambitious yet: 166 D-hosts with a little over 1TB of XML stored on each, plus a handful of E-hosts to manage incoming queries. Each host was equipped with two dual-core AMD Opteron CPUs, 16-GB RAM and RedHat Enterprise Linux. Storage was a shared SAN, connected via Fibrechannel.

The most important proof point for this cluster was to show that we could scale out a cluster to over 150 hosts. We demonstrated this for the customer at an early stage, when 80 hosts had been assembled and configured as a cluster. We loaded content on each host, and showed that we could scale up the number of

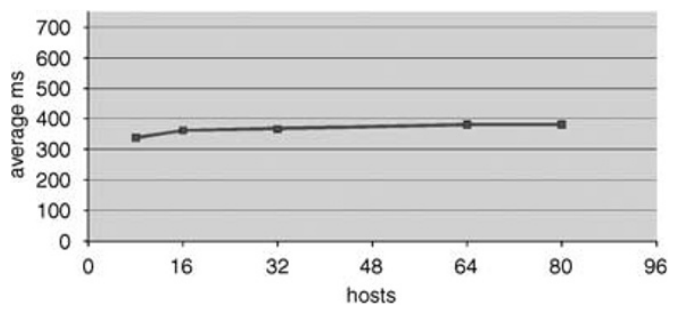

Figure 6: The same workload was tested with clusters of 8-80 hosts. Query response times were unchanged as the number of hosts increased

hosts in the cluster without materially affecting query performance. As the figure shows, there was virtually no difference in performance as we scaled the cluster up from 16 to 80 hosts.

\section{CONCLUSIONS}

This is not the end of the story. Hardware is still becoming cheaper and faster every year. Next year, 8-16 CPU cores will become more common, and 32-64 GB RAM will become more affordable. Storage prices will continue to fall, and densities will continue to increase.

New application deployments will be able to support more concurrent queries, and more content, without costing more money. All this adds up to bigger, faster XQuery.

Our engineers continue to enhance and optimize the product, too. MarkLogic Server 3.2 shipped in May, adding new index options, new configuration tuning options and improved performance on existing queries. As our customers build these new possibilities into their code, we expect an exciting new crop of content-driven applications. Some of these applications will push content-server scalability to new heights. We see hundreds of TB on the horizon, and petabytes in the not-too-distant future.

\section{References}

1 Ciarlone, L. (2006) 'The reality of Web 2.0: O'Reilly media's SafariU leads by example', Gilbane Content Technology Works, http://gilbane. $\mathrm{com} /$ case_studies/oreilly_case_study.html.

2 Coutts, D. (2005) 'Modeling rich content for reuse', Journal of Digital Asset Management, Vol. 1, No. 4, pp. 271-278

3 Stonebreaker, M. and Çetintemel, U. (2005) ““One Size Fits All": An idea whose time has come and gone', 21st International Conference on Data Engineering (ICDE 2005), National Center of Sciences, Tokyo, Japan. 